\title{
Influence of Trapezoidal Shapes and Cutout Sizes on the Buckling Behaviour of Composite Laminates Under Thermally Induced Loads
}

\author{
Maharudra $^{1,3 *}$ (D), T. Rajanna ${ }^{2}$ (D), Bheemsha Arya $^{1}$ (D) \\ ${ }^{1}$ Department of Mechanical Engineering, B.M.S College of Engineering, Bengaluru-560019, Karnataka, India. E-mail: \\ rudresh2100@gmail.com, arya.mech@bmsce.ac.in \\ ${ }^{2}$ Department of Civil Engineering, B.M.S College of Engineering, Bengaluru-560019, Karnataka, India. E-mail: t.rajanna@gmail.com, \\ ${ }^{3}$ Department of Mechanical Engineering, Government Polytechnic College, Bagepalli -561207, Karnataka, India. E-mail: \\ rudresh2100@gmail.com,
}

${ }^{*}$ Corresponding author

https://doi.org/10.1590/1679-78256331

\begin{abstract}
Trapezoidal laminated plates with cutouts are commonly found in many engineering field, especially in aerospace structures. In many cases these plates are subjected to various harsh environmental conditions during its service life. Thermally induced load is the one which seriously affect the buckling characteristics of the structural components. The study presents the effect of rise in temperature on the thermal buckling characteristics of trapezoidal laminated composite plates with and without cutouts by using finite element technique. In order to model the plate, a 9-noded heterosis plate element has been used by incorporating the effect of shear deformation. By correlating present findings with the available literature, the effectiveness of the present formulation is verified. The computer program FORTRAN language has been developed to investigate the effect of different parameters such as trapezoidal shapes, cutout offsets, plate aspect ratio, ply-orientations, different thickness and plate edge conditions. The influence of each parameter on the thermal buckling behavior is well investigated through this work.
\end{abstract}

\section{Keywords}

Trapezoidal shape, cutout offset, composite laminates, heterosis element, thermal loads, cutout size.

\section{Graphical Abstract}
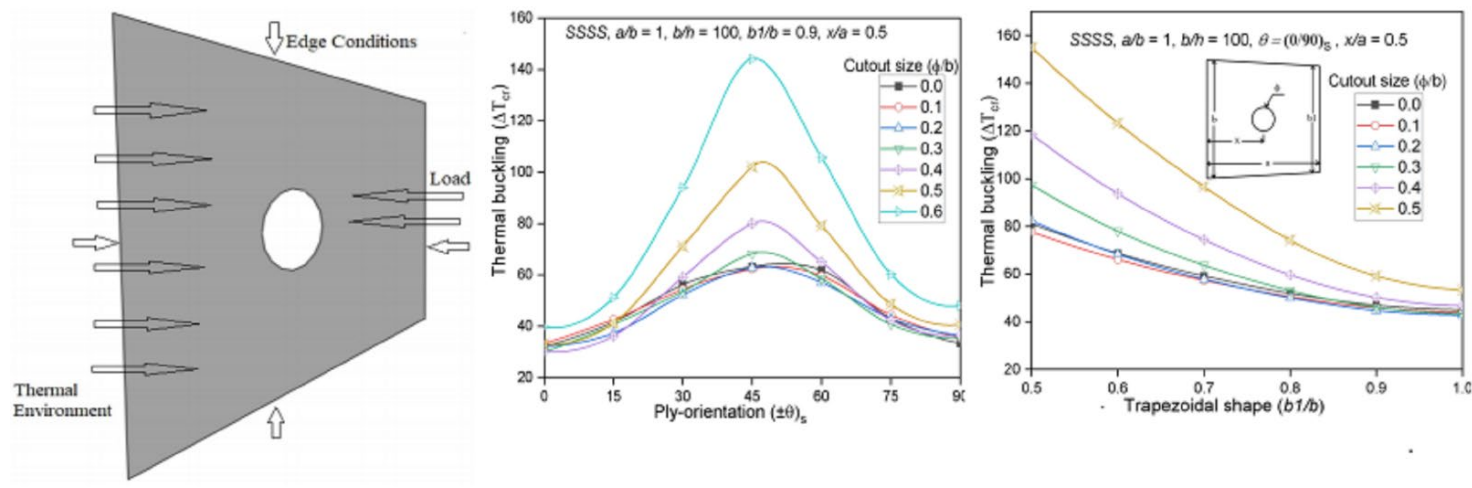

Received November 11, 2020. In revised form February 03, 2021. Accepted February 14, 2021. Available online February $22,2021$. https://doi.org/10.1590/1679-78256331

CC) Latin American Journal of Solids and Structures. ISSN 1679-7825. Copyright C 2021. This is an Open Access article distributed under the terms of the Creative
Commons Attribution License, which permits unrestricted use, distribution, and reproduction in any medium, provided the original work is properly cited. 


\section{INTRODUCTION}

Advances in the material science have resulted in a remarkable rise in the allowable material stress level. It is more so in the case of recently developed light-weight and high-strength composite materials. In most of the cases, cut-outs or openings are provided within the laminated plates to satisfy functional requirements, such as easy access for inspection, passing hydraulic lines, fuel lines and electrical lines, and often simply reducing the structure's total weight. The wide usage and application of such materials, many a time subjected to various kinds of harsh environmental conditions during its service life. The elevated temperature is the one which seriously affect the buckling characteristics of laminated plates, especially the geometrically discontinued plates. The in-plane thermal stress distribution inside the plate may be complicated by these discontinuities in the plates, whereby the stress field is not only non-uniform, the existence of stresses may also vary in different regions, resulting in a loss of stability at an extremely low level of stresses. Therefore, the complete knowledge of multiple formed plates with cut-outs is most necessary in order to prevent the premature failure and to use their full strength, which can be tremendously explored in the area of thin walled structures. The fibre - reinforced composite structures are vulnerable to elevated temperature loads during the operational life and which leads to considerable degradation of strength and other damaging effects.

Biswal et al. (2016) investigated the effect of hygro-thermal loading on buckling behaviour of laminated composite shell by adopting the finite element method (FEM). A quadratic iso-parametric eight-noded shell element with first order shear deformation theory (FSDT) was used for modeling the shell. Also, experiment was conducted to support these results. In order to ascertain the parametric instability of laminated plates subjected to periodic dynamic loading in hygrothermal environments, Rath and Sahu (2013) adopted the finite element modelling approach. Theoretical and analytical investigations based on the finite element factors were performed by Panda et al. (2013) to study hygro-thermal effects on free vibration of delaminated woven composite fibre plates. Rath and Sahu (2013) studied the vibration characteristics of woven fibre glass/epoxy delaminated composite plates subjected to higher temperatures and humidity. They employed eight-noded quadratic iso-parametric element with 5-degrees of freedom per node and first order shear deformation theory (FSDT) has been used for modelling the plate. Kar et al. (2016) studied the thermal buckling behaviour of shear deformable functionally graded single/doubly curved shell plates with temperature-dependent (TD) and temperature-independent (TID) characteristics. Na and Kim (2004) investigated the thermal buckling behaviour of the FG plate by using finite element method (FEM). Abdelhak et al. (2015) examined the thermal buckling behaviour of Functionally Graded Materials (FGMs) using four variable theory in the $\mathrm{n}^{\text {th }}$ order. Zenkour (2012) used a sinusoidal principle to investigate hygrothermal influence on the buckling behaviour of angle-ply laminated composites. To present hygro-thermal effects on the buckling behaviour of laminated composite plates, Ram and Sinha (1991) have used the finite element method (FEM). The effect of hygro-thermal environment on post-buckling behaviour of laminated plates based on Reddy's higher order plate theory (HOPT) has been studied by Shen (2001). Abida et al. (2020) have extensively examined the hygro-mechanical combined effect on correlation coefficient analysis of flax yarns and flax/epoxy composites. Abdelmalek et al. (2017) observed the hygrothermal effects of composite plate free vibration behaviour using node n-order shear deformation theory. Gayen and Roy (2013) conducted investigations on the hygro-thermal effects of stress analysis of tapered laminated composite structural beams by considering finite element (FE) techniques. Using higher-order theory Patel et al. (2002) did research on hygrothermal effects on structural design of thick composite laminates. Sahin (2005) investigated the impact of thermal buckling behaviour of hybrid angle-ply laminated composite plate with a hole. The thermal buckling analysis using a simplified higher order shear deformation theory (HSDT) for cross-ply laminated plates has been investigated by Chikh et al. (2017). Chandrashekhara (1992) has been analysed the thermal buckling behaviour of laminated plate using shear flexible finite element technique. Shu and Sun (1994) studied the thermal buckling behaviour of cross-ply simply supported plates using higher order shear deformation theory (HSDT). Mansouri and Shariyat (2014) have studied the thermal buckling predictions of heterogeneous orthotropic plates using the new variant of high-order theories. Sam et al. (2012) examined the buckling analysis of functionally graded plates under thermal loadings using the finite strip method. Chen et al. (2011) has done the research on the thermally induced buckling of functionally graded hybrid composite plates. Shariyat (2007) has done the study on thermal buckling analysis of rectangular composite plates with Temperature-dependent (TD) Properties based on a layerwise theory. Noor and Burton (1992) has used the three-dimensional solutions for the analysis of thermal buckling of multilayered anisotropic plates. Carrera (2005) has investigated the transverse normal strain effects on thermal stress analysis of homogeneous and layered plates.

From the above literature study, it can be concluded that the vibration and buckling behaviour of laminated plates subjected to hygrothermal loads is addressed in a wide amount. Very few literature deals with the effect of thermal loads with centrally located circular cutout, but they are restricted to square/rectangular laminated plates. In the literature, the thermal buckling behaviour of the trapezoidal plate is very scanty under thermal loads. According to the best of 
author's knowledge, no study is found in the literature which contributes to the effect of cutout sizes and its offset on the thermal buckling behaviour of the trapezoidal laminate composite plates. Therefore, by taking into account various parameters such as trapezoidal shapes, cutout offsets, ply-orientation, plate edge condition, plate aspect ratio and width to thickness ratio, an effort is made to study the impact of trapezoidal shape and the cutout offsets on the thermal buckling behaviour of trapezoidal plate through this work by adopting nine-noded heterosis element.

\section{MATHEMATICAL FORMULATION}

The basic configuration of the problem considered here is a trapezoidal shaped plate with a circular cutout, as illustrated in Figure 1 along with the displacement coordinates for the $x-, y$-, and $z$-axes.

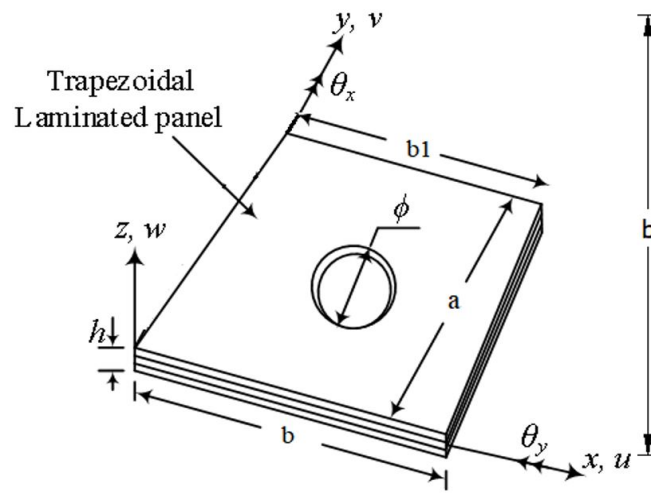

(a)

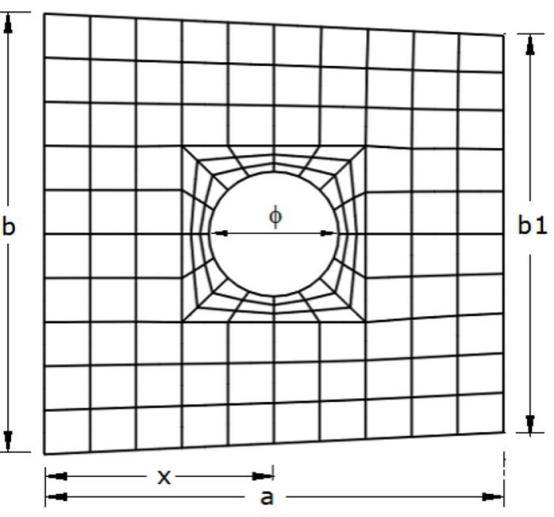

(b)

Figure 1 (a) Geometry of the plate with its co-ordinate system. (b) Detailed mesh pattern.

The trapezoidal laminated plate consists of $N$ layers and each layer is of thickness $t_{n}$, so the laminate total thickness $h$ is given by $h=\sum_{n=1}^{N} t_{n}$. The laminate's lateral and longitudinal measurements are $b$ and $a$ are exposed to a uniform temperature rise $\Delta T$. The linear stress-strain relationship for each layer is expressed with $x, y$ axes and has the form.

$$
\begin{aligned}
& \left\{\begin{array}{l}
\sigma_{x x} \\
\sigma_{y y} \\
\tau_{x y}
\end{array}\right\}_{n}=\left[\begin{array}{lll}
\overline{\bar{Q}}_{11} & \overline{\bar{Q}}_{12} & \overline{\bar{Q}}_{16} \\
\overline{\bar{Q}}_{12} & \overline{\bar{Q}}_{22} & \overline{\bar{Q}}_{26} \\
\overline{\bar{Q}}_{16} & \overline{\bar{Q}}_{26} & \overline{\bar{Q}}_{66}
\end{array}\right]_{n}\left\{\begin{array}{l}
\varepsilon_{x x}-\alpha_{x x} \Delta T \\
\varepsilon_{y y}-\alpha_{y y} \Delta T \\
\gamma_{x y}-\alpha_{x y} \Delta T
\end{array}\right\}_{n}, \\
& \left\{\begin{array}{l}
\tau_{y z} \\
\tau_{x z}
\end{array}\right\}=\left[\begin{array}{ll}
\overline{\bar{Q}}_{44} & \overline{\bar{Q}}_{45} \\
\overline{\bar{Q}}_{45} & \overline{\bar{Q}}_{55}
\end{array}\right]\left\{\begin{array}{l}
\gamma_{y z} \\
\gamma_{x z}
\end{array}\right\},
\end{aligned}
$$

where $\sigma_{x x}, \sigma_{y y}, \tau_{x y}, \tau_{y z}$ and $\tau_{x z}$ are the stress components, $\Delta T$ is the uniform temperature rise, $\alpha_{x x}$ and $\alpha_{y y}$ are the thermal expansion coefficients in $x$ and $y$ axes, respectively and $\alpha_{x y}$ is the thermal coefficient of shear and are given by

$$
\begin{aligned}
& \alpha_{x x}=\alpha_{1} \cos ^{2} \theta+\alpha_{2} \sin ^{2} \theta, \\
& \alpha_{y y}=\alpha_{2} \cos ^{2} \theta+\alpha_{1} \sin ^{2} \theta, \\
& \alpha_{x y}=2\left(\alpha_{1}-\alpha_{2}\right) \sin \theta \cos \theta .
\end{aligned}
$$

Here $\alpha_{2}$ and $\alpha_{1}$ are the coefficients of thermal expansions in transverse and longitudinal of the fibers, respectively.

The Reissner-Mindlin's plate theory is employed in this study based on the assumption that the mid-plane normal remains straight but not necessarily perpendicular to the axis after deflection. The displacement field are stated as (Maharudra and Rajanna 2020), 


$$
\begin{aligned}
& U(x, y, z)=u_{0}(x, y)+z \theta_{x}(x, y), \\
& V(x, y, z)=v_{0}(x, y)+z \theta_{y}(x, y), \\
& W(x, y, z)=w_{0}(x, y),
\end{aligned}
$$

where $\vartheta_{y}$ and $\vartheta_{x}$ are rotations of the mid-plane with respect to $y$ and $x$ axes respectively and $u_{0}, v_{0}$ and $w_{0}$ are the displacements an arbitrary point $(x, y)$ on the mid-plane.

The bending strains and shear strains at any point of the laminate are given by

$$
\begin{aligned}
& \left\{\begin{array}{l}
\varepsilon_{x x} \\
\varepsilon_{y y} \\
\gamma_{x y}
\end{array}\right\}=\left|\begin{array}{c}
\frac{\partial \boldsymbol{u}_{\mathrm{o}}}{\partial x} \\
\frac{\partial \boldsymbol{v}_{\mathrm{o}}}{\partial y} \\
\frac{\partial \boldsymbol{u}_{\mathrm{o}}}{\partial y}+\frac{\partial \boldsymbol{v}_{\mathrm{o}}}{\partial x}
\end{array}\right|+z\left|\begin{array}{c}
\frac{\partial \boldsymbol{\theta}_{x \mathrm{o}}}{\partial \boldsymbol{x}} \\
\frac{\partial \boldsymbol{\theta}_{y}}{\partial y} \\
\frac{\partial \boldsymbol{\theta}_{x}}{\partial y}+\frac{\partial \boldsymbol{\theta}_{y}}{\partial \boldsymbol{x}}
\end{array}\right|, \\
& \left|\begin{array}{l}
\gamma_{y z} \\
\gamma_{x z}
\end{array}\right|=\left|\begin{array}{l}
\frac{\partial w_{0}}{\partial y}+\theta y \\
\frac{\partial w_{0}}{\partial y}+\theta x
\end{array}\right|,
\end{aligned}
$$

where $\varepsilon_{x x}$ and $\varepsilon_{y y}$ denotes the bending strains and $\gamma_{x y}, \gamma_{y z}$, and $\gamma_{x z}$ denotes the shear strains at any point of the laminated plate.

The resultant forces $N_{x x}, N_{y y}$ and $N_{x y}$, moments $M_{x x} M_{y y}$ and $M_{x y}$ and shearing forces $Q_{x x}, Q_{y y}$ per unit length of the plate are given as

$$
\begin{aligned}
& {\left[\begin{array}{ll}
N_{x x} & M_{x x} \\
N_{y y} & M_{y y} \\
N_{x y} & M_{x y}
\end{array}\right]=\int_{-h / 2}^{h / 2}\left\{\begin{array}{l}
\sigma_{x x} \\
\sigma_{y y} \\
\tau_{x y}
\end{array}\right\}(1, z) d z} \\
& \left\{\begin{array}{l}
Q_{x x} \\
Q_{y y}
\end{array}\right\}=\int_{-h / 2}^{h / 2}\left\{\begin{array}{l}
\tau_{x z} \\
\tau_{y z}
\end{array}\right\} d z
\end{aligned}
$$

The thermal load total potential energy $\pi$ of a laminated plate is equated to

$$
\pi=U_{b s}+U_{s s}+\Gamma
$$

where $U_{b s}, U_{s s}$ and $\Gamma$ denotes the strain energy of bending, strain energy of shear and potential energy, respectively of in-plane loadings due to the alteration in temperature:

$$
\begin{aligned}
& U_{b s}=1 / 2 \int_{-h / 2}^{h / 2}\left[\iint_{r}\left(\sigma_{x} \varepsilon_{x x}+\sigma_{y} \varepsilon_{y y}+\tau_{x y} \gamma_{x y}\right) d \Lambda\right] d z, \\
& U_{s s}=1 / 2 \int_{-h / 2}^{h / 2}\left[\iint_{r}\left(\tau_{x z} \gamma_{x z}+\tau_{y z} \gamma_{y z}\right) d \Lambda\right] d z, \\
& \Gamma=1 / 2 \iint_{r}\left[\bar{N}_{1}\left(\partial w_{0} / \partial x\right)^{2}+\bar{N}_{2}\left(\partial w_{0} / \partial y\right)^{2}\right. \\
& \left.+2 \bar{N}_{12}(\partial w / \partial x)(\partial w / \partial y)\right] d \Lambda-\int_{\partial r}\left(\bar{N}_{n}^{b} u_{n}^{0}+\bar{N}_{s}^{b} u_{s}^{0}\right) d s,
\end{aligned}
$$

where $d \Lambda=d x d y, r$ is the plate region excluding hole. $\bar{N}_{n}^{b}, \bar{N}_{s}^{b}$ are in-plane loads applied on the boundary.

To achieve equilibrium, the potential energy $\pi$ must be stationary. The equilibrium equations of the angle-ply laminated plate subjected to temperature change can be derived from the variational principle through the use of stressstrain and strain-displacement relations. One may obtain these equations by $\partial \pi=0$ (Bathe 2014). 


\subsection{FE formulation}

A computer program by using FORTRAN language has been developed to work out buckling problems. The different participating structural matrices are obtained by assembling each individual element level matrices by using skyline technique. Eigenvalues and Eigenvectors are obtained by using subspace iteration technique (Bathe 2014). In the computer program, selective integration scheme, in which $3 \times 3$ gauss rule for in plane as well as bending terms and $2 \times$ 2 gauss rule for shear terms has been incorporated to generate element elastic stiffness matrix without any shear locking. Similarly, a full integration scheme (i.e., $3 \times 3$ gauss rule) has been used for both geometric stiffness as well as mass matrices. Figure 2 shows the schematic example of various types of elements. The 9-noded heterosis element as shown in Figure 2 has been used in the discretization of the trapezoidal plate.
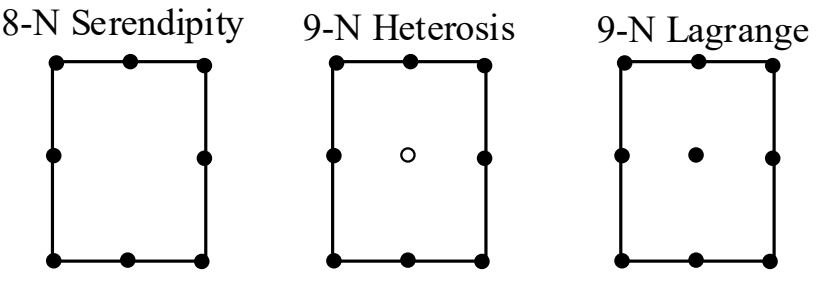

- Node with $u, v, w, \theta_{x}, \theta_{y}$ degrees of freedom

○ Node with $u, v, \theta_{x}, \theta_{y}$ degrees of freedom

Figure 2 Schematic illustration of different types of elements.

The 9-noded heterosis element is generated by incorporating 9-noded Lagrangian shape functions for $u, v, \vartheta_{x}, \vartheta_{y}$ and 8-noded serendipity shape functions for $w$ degrees of freedom. A 9-noded heterosis plate element has been employed in the current research to investigate the buckling behaviour of the trapezoidal plate. This type of element exhibits high level accuracy as compared to that of 8-noded serendipity and 9-noded Lagrangian elements (Rajanna et al. 2016).

By the use of minimum potential energy rule, the stiffness matrix of the plate is achieved. Bending stiffness [ $\left.K_{b s}\right]$, shear stiffness $\left[K_{s s}\right]$ and geometric stiffness $\left[K_{g s}\right]$ can be represented as:

$$
\begin{aligned}
& {\left[K_{b s}\right]=\int_{\Lambda}\left[B_{b s}\right]^{T}\left[D_{b s}\right]\left[B_{b s}\right] d \Lambda,} \\
& {\left[K_{s s}\right]=\int_{\Lambda}\left[B_{s s}\right]^{T}\left[D_{s s}\right]\left[B_{s s}\right] d \Lambda,}
\end{aligned}
$$

and

$$
\left[K_{g s}\right]=\int_{\Lambda}\left[B_{g s}\right]^{T}\left[D_{g s} \llbracket B_{g s}\right] d \Lambda
$$

where,

$$
\begin{aligned}
& {\left[D_{b s}\right]=\left[\begin{array}{ll}
A_{i j} & B_{i j} \\
B_{i j} & D_{i j}
\end{array}\right],} \\
& {\left[D_{s s}\right]=\left[\begin{array}{cc}
k_{1}^{*} A_{44} & 0 \\
0 & k_{2}^{*} A_{45}
\end{array}\right],} \\
& {\left[D_{g s}\right]=\left[\begin{array}{ll}
\bar{N}_{1} & \bar{N}_{12} \\
\bar{N}_{12} & \bar{N}_{2}
\end{array}\right]}
\end{aligned}
$$




$$
\begin{aligned}
& \left(A_{i j}, B_{i j}, D_{i j}\right)=\int_{-h / 2}^{h / 2} \bar{Q}_{i j}\left(1, z, z^{2}\right) d z \quad(i, j=1,2,6) \\
& \left(A_{44}, A_{55}\right)=\int_{-h / 2}^{h / 2}\left(\overline{\bar{Q}}_{44}, \overline{\bar{Q}}_{45}\right) d z .
\end{aligned}
$$

Here $\left\lfloor A_{i j}\right\rfloor,\left\lfloor B_{i j}\right\rfloor$ and $\left\lfloor D_{i j}\right\rfloor$ represents extension, extension-bending and bending stiffness respectively and $k_{1}^{*}=k_{2}^{*}=5 / 6$ represents the shear correction factors.

\subsection{Governing Equations}

After assembling the elastic stiffness and the geometric stiffness matrices, the corresponding eigenvalue problem can be solved by using any standard extraction procedures (Muddappa et al. 2021).

$$
\left.\llbracket \bar{K}_{0}\right]-\lambda_{c r}\left[\bar{K}_{0 g} \rrbracket\left\{\begin{array}{l}
u_{i} \\
v_{i} \\
w_{i}
\end{array}\right\}=0\right.
$$

where

$$
\begin{aligned}
& {\left[\bar{K}_{0}\right]=\left[K_{b s}\right]+\left[K_{s s}\right]} \\
& -\lambda_{c r}\left[\bar{K}_{0 g}\right]=\left[K_{g s}\right]
\end{aligned}
$$

The product of $\lambda_{c r}$ and the initial guest value $\Delta T$ is the critical buckling temperature $T_{c r}$, that is (Sahin 2005)

$$
\Delta T_{c r}=\lambda_{c r} \Delta T
$$

\section{DESCRIPTION OF THE PROBLEM}

The trapezoidal laminated plate considered in this research is thin in nature with $b / h=100$ and, unless otherwise stated, the thermo-elastic properties of the material that are considered for E-glass/epoxy are shown in Table 1 and Table 2.

Table 1 Material properties used for numerical solutions at different temperature are (Sairam, 1991).

\begin{tabular}{cccccccc}
\hline \multirow{2}{*}{ Elastic Moduli (GPa) } & \multicolumn{5}{c}{ Temperature $\boldsymbol{T}(\mathbf{K})$} \\
\cline { 2 - 7 } & $\mathbf{3 0 0}$ & $\mathbf{3 2 5}$ & $\mathbf{3 5 0}$ & $\mathbf{3 7 5}$ & $\mathbf{4 0 0}$ & $\mathbf{4 2 5}$ \\
\hline$E_{1}$ & 130.0 & 130.0 & 130.0 & 130.0 & 130.0 & 130.0 \\
$E_{2}$ & 9.50 & 8.50 & 8.00 & 7.50 & 7.00 & 6.75 & 4.50 \\
$G_{12}$ & 6.00 & 6.00 & 5.50 & 5.00 & 4.75 & 4.50 \\
\hline
\end{tabular}

Table 2 Material properties of the composite laminate used for the analysis.

\begin{tabular}{cc}
\hline Mechanical properties & Value/Notation \\
\hline Modulus of elasticity in direction 1 & $E_{1}$ \\
Modulus of elasticity in direction 2 & $E_{2}$ \\
Shear modulus in fiber direction $-G_{12}, G_{13}$ & $G_{12}=G_{13}$ \\
Shear modulus in lateral direction $-G_{23}$ & $G_{23}=0.5 G_{13}$ \\
Poisson's ration - $\mu_{12}$ & 0.30 \\
Coefficients of thermal expansions $-\alpha_{1}$ & $-0.3 \mathrm{E}^{-6} / \mathrm{K}$ \\
Coefficients of thermal expansions $-\alpha_{2}$ & $28.1 \mathrm{~K}^{-6} / \mathrm{K}$ \\
\hline
\end{tabular}


In the string of any support conditions, the notations SSSS and CCCCC indicates simply support and clamped conditions respectively. The displacement based boundary conditions considered in this work are as follows (Chandra et al. 2020):

(1) Simply supported condition (SSSS):

$$
\begin{aligned}
& \text { At } x=0, a ; u_{0}=w_{0}=\vartheta_{y}=0 \\
& \text { At } y=0, b ; v_{0}=w_{0}=\vartheta_{x}=0
\end{aligned}
$$

(2) Clamped condition (CCCC):

$$
\begin{aligned}
& \text { At } x=0, a ; u_{0}=v_{0}=w_{0}=\vartheta_{x}=\vartheta_{y}=0 \\
& \text { At } y=0, b ; u_{0}=v_{0}=w_{0}=\vartheta_{x}=\vartheta_{y}=0
\end{aligned}
$$

\section{RESULTS AND DISCUSSIONS}

There are several approaches for solving eigenvalue problems. In this analysis, the subspace iteration technique is applied to get numerical solutions to the problem in order to obtain the eigenvalues and eigenvectors. The laminated trapezoidal composites with symmetrically bonded angle-ply and cross-ply plates are considered and whose thickness, $b / h=100$ unless otherwise specified.

\subsection{Comparison and Convergence study}

In order to check the validity of the mesh to be considered for relatively accurate converged outcomes, the convergence study is most important. A $10 \times 10$ mesh size is found to be so well converged in this study and throughout this work the same mesh size is chosen. But at the other hand, the comparison study is also most relevant to determine the accuracy of the convergence study established for buckling of laminated plate to validate the current finite element

\begin{tabular}{|c|c|c|c|c|c|c|}
\hline \multirow{4}{*}{$a / b$} & \multicolumn{5}{|c|}{ Critical buckling Temperature $\left(\alpha T_{c r} \times 10^{-4}\right)$} & \multirow{3}{*}{$\begin{array}{c}\text { Chandrashekhara (1992) } \\
\text { Mess size }\end{array}$} \\
\hline & \multicolumn{5}{|c|}{ Present } & \\
\hline & \multicolumn{5}{|c|}{ Mess size } & \\
\hline & $4 \times 4$ & $5 \times 5$ & $10 \times 10$ & $15 \times 15$ & $20 \times 20$ & $4 \times 4$ \\
\hline 0.25 & 0.6731 & 0.6725 & 0.6720 & 0.6720 & 0.6720 & 0.6727 \\
\hline 0.50 & 0.7918 & 0.7912 & 0.7906 & 0.7905 & 0.7905 & 0.7913 \\
\hline 0.75 & 0.9897 & 0.9889 & 0.9882 & 0.9881 & 0.9881 & 0.9890 \\
\hline 1.00 & 1.2667 & 1.2657 & 1.2648 & 1.2647 & 1.2646 & 1.2657 \\
\hline 1.25 & 1.6227 & 1.6214 & 1.6202 & 1.6201 & 1.6201 & 1.6234 \\
\hline 1.50 & 2.0577 & 2.056 & 2.0546 & 2.0544 & 2.0543 & 2.0561 \\
\hline 1.75 & 2.5715 & 2.5695 & 2.5676 & 2.5674 & 2.5674 & 2.5696 \\
\hline 2.00 & 3.1641 & 3.1615 & 3.1593 & 3.1590 & 3.1589 & 3.1617 \\
\hline 2.25 & 3.8354 & 3.8322 & 3.8295 & 3.8292 & 3.8291 & 3.8324 \\
\hline 2.50 & 4.5850 & 4.5813 & 4.5780 & 4.5777 & 4.5776 & 4.5817 \\
\hline 2.75 & 5.4132 & 5.4088 & 5.4050 & 5.4046 & 5.4044 & 5.4096 \\
\hline 3.00 & 6.3193 & 6.3152 & 6.3098 & 6.3093 & 6.3091 & 6.3144 \\
\hline
\end{tabular}
software. The comparisons of the current results gathered against the existing results are shown in Table 3.

Table 3 Comparison of the temperature buckling loads of simply supported (SSSS) isotropic plate. $E=1.0 \times 10^{9} \mathrm{~Pa}, \mu=0.3, a / h=100$, $a / b=1, a=254, \alpha_{0}=1.0 \mathrm{E}^{-6}$

It is observed from the above convergence study that, the mesh size, $10 \times 10,15 \times 15$ and $20 \times 20$, are almost having the same critical buckling temperature, hence in this numerical analysis $10 \times 10$ mesh size is considered throughout the problem. 


\subsection{Case studies on buckling behaviour of trapezoidal plate under uniform thermal loads}

In this section, the thermal buckling behaviour of trapezoidal composite laminates are examined by considering various parameter such as different ply orientations, cutout sizes, cutout offsets, plate aspect ratio, thickness ratio and different trapezoidal shape ratios, etc. The term trapezoidal indicates when the $b_{1}$ values changes from 0.5 to 1 , where $b_{1}$ reaches $b$, it will become square plate. Both simply supported and clamped edge conditions are employed to investigate the thermal buckling behaviour laminated plate.

\subsubsection{Thermal buckling behaviour under different ply-orientations and cutout sizes.}

The thermal buckling behaviour of trapezoidal plates with different ply-orientations and cutout sizes are considered. The cutout offset ratio $(x / a)$ taken as 0.5 with thickness ratio $(b / h)=100$ under the action of unit temperature is studied in this section and the results are shown in Figure 3. The plate considered here is trapezoidal in shape and all four sides are simply supported and clamped edge conditions. It is evident from Figure 3 (a) and Figure 3 (b) that the thermal buckling $\left(\Delta T_{c r}\right)$ of the plate increases with the increase in ply-angle and found highest at ply-orientation of $( \pm 45)_{s}$ thereafter decreases with the further increase in ply-angle. It is important to note that the value of $\Delta T_{c r}$ significantly higher when the $\phi / b=0.6$. As the cutout size increases, the thermal stress distribution within the vicinity of the cutout moves towards the stiffer region of the plate and this may be the reason for the increasing thermal buckling resistance with respect to the cutout size. It is also observed from the graph that, the thermal bucking resistance of the clamped edge conditions is higher as compared with the simply supported edge condition for all ply-orientation. It is worthwhile to mention that angle-ply laminates with higher cutout size shows higher buckling resistance.

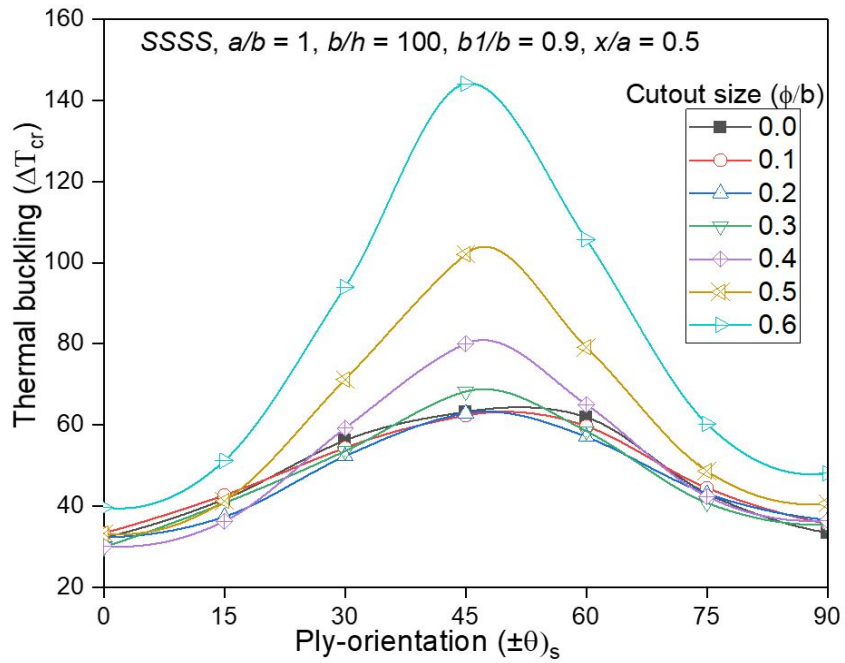

(a)

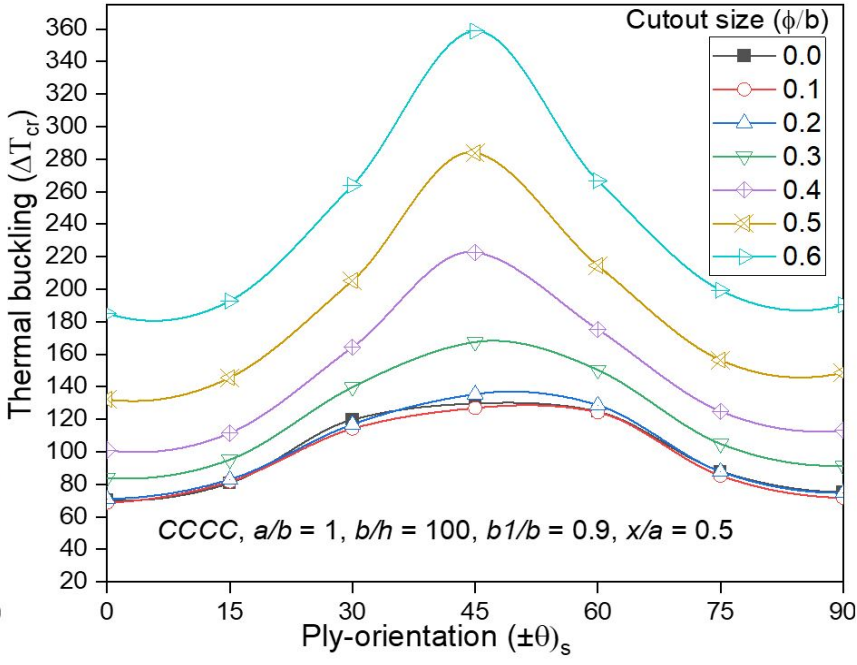

(b)

Figure 3 Thermal buckling behaviour under (a) SSSS and (b) CCCC edge conditions.

\subsubsection{Thermal buckling behaviour under various trapezoidal shapes with cutouts.}

Influence of trapezoidal shape ratio $\left(b_{1} / b\right)$ and the cutout sizes on the critical thermal buckling $\left(\Delta T_{c r}\right)$ behaviour have been studied and the results are shown in Figure 4 . Simply supported boundary condition with cutout offset ( $x / a$ ) 0.5 is considered here. From the Figure 4 (a) and Figure 4 (b), it is cleared that the thermal buckling resistivity of the plate progressively decreases as the trapezoidal shape increases in both cross ply laminates as well as angle play laminates. Further, it is observed that there is much variation of the thermal buckling behaviour in case of angle ply laminates as compared with the cross ply laminates. As the cutout size increases, the thermal buckling resistivity of the plate will also increase, this is due to thermal stress distribution within the vicinity of the cutout moves towards the stiffer region of the plate. And also as the trapezoidal shape ratio increases irrespective of the $\phi / b$ ratio, thermal bucking resistance increases and found peak at trapezoidal shape ratio $\left(b_{1} / b\right)$ is 0.5 . This increasing buckling strength may be due to the increase in stiffness of the plate due to the reduction of span length. 


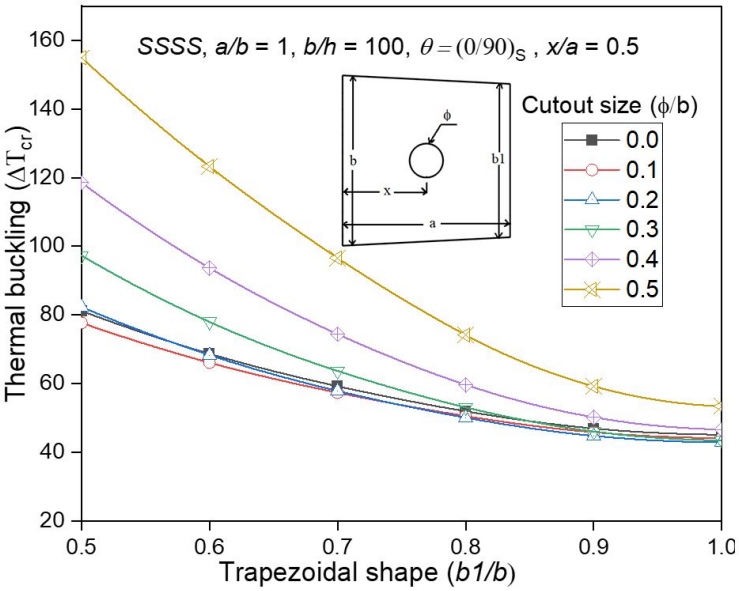

(a)

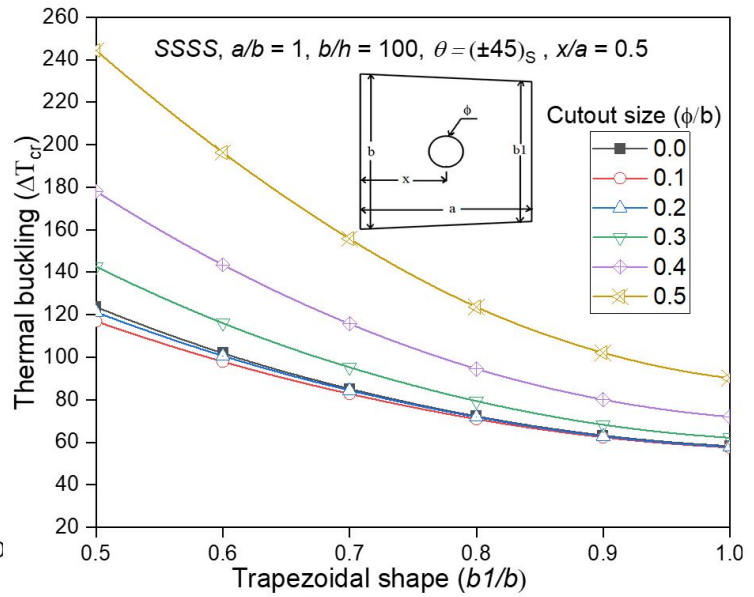

(b)

Figure 4 Thermal buckling behaviour under different trapezoidal shapes (a) cross ply and (b) angle ply

\subsubsection{Impact of cutout offsets and trapezoidal shapes on thermal buckling behaviour.}

Influence of cutout offsets $(x / a)$ and the trapezoidal shapes on the critical thermal buckling $\left(\Delta T_{c r}\right)$ behaviour has been studied and the results are illustrated in Figure 5. The cutout offset ratios $(x / a)$ which are considered are $0.2,0.4$, 0.6 and 0.8 of the total length of the plate. In order to compare the results, a symmetric angle ply and cross ply schemes are considered with cutout size 0.2 under simply supported edge conditions. It is evident from Figure 5 (a) and Figure 5 (b) that the buckling resistance of plate decreases with the increase in trapezoidal shapes. Thermal buckling resistance is quite high in case of the cutouts which are located at the edges of the plate. As the cutout offsets towards the centre of the plate, the thermal buckling resistance observed is quite lower. As usual as explained in the above sections, the thermal buckling resistance is higher at trapezoidal shape ratio $\left(b_{1} / b\right)$ is 0.5 and minimum at trapezoidal shape ratio $\left(b_{1} / b\right)$ is 1.0. The thermal buckling stability decreases gradually when cutout move towards the higher stiffness zones. However, this behaviour is same in cross ply laminates, and not found much difference in the thermal buckling stability as the cutout offsets. Therefore, it is clear from the analysis that, angle ply laminated plate with $( \pm 45)_{s}$ is having good ability to withstand the higher thermal buckling load.

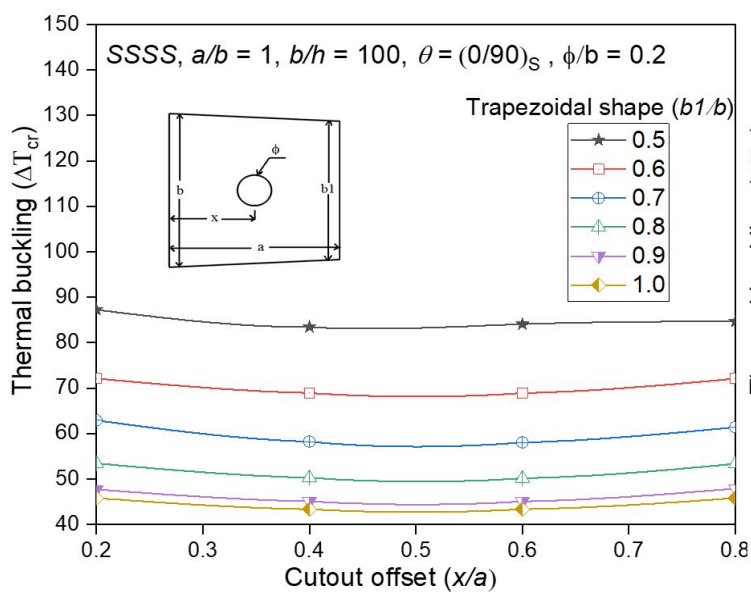

(a)

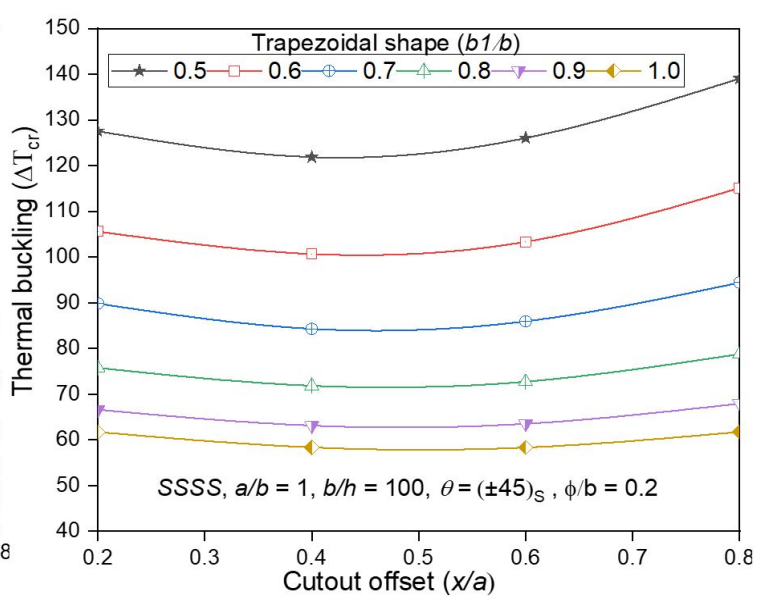

(b)

Figure 5 Thermal buckling behaviour under different cutout offsets (a) cross ply and (b) angle ply.

\subsubsection{Impact of different thickness and cutout sizes on thermal buckling behaviour.}

The same research is carried out to investigate the effect of different thickness and cutout sizes on the thermal buckling behaviour by considering two different ply configurations. Simply supported edge condition is considered with cutout offset 0.5 . Trapezoidal shape ratio $\left(b_{1} / b\right)$ considered here is 0.5 with various thickness ratios $(b / h)$. The results are elaborated in Figure 6. It is very clear from the Figure 6 (a) and Figure 6 (b) that the value of $\Delta T_{c r}$ decreases with the increase in plate thickness ratio $(b / h)$ with respective to cutout sizes. As the thickness decreases, the laminated plate becomes thin, and hence reduces the strength. This leads to decrease in the stability of the laminated plate. It is clear 
from the analysis that, the highest thermal buckling stability is seen in the case of angle ply laminates i.e $( \pm 45)_{s}$. As the thickness ratio $(b / h)$ and cutout sizes increases, there is no much of the increase in the thermal buckling stability found, wherein the value of $\Delta T_{c r}$ found to be remain constant for thickness ratios $(b / h)$ beyond 40.

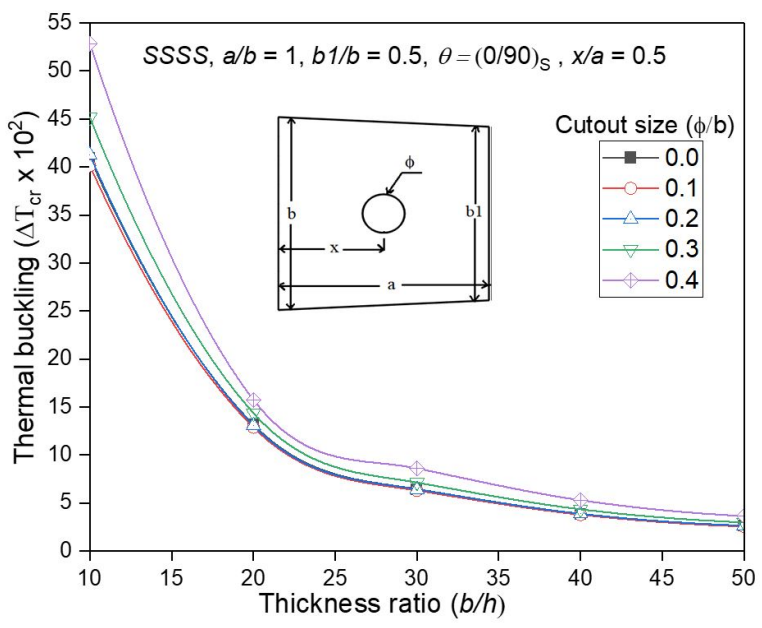

(a)

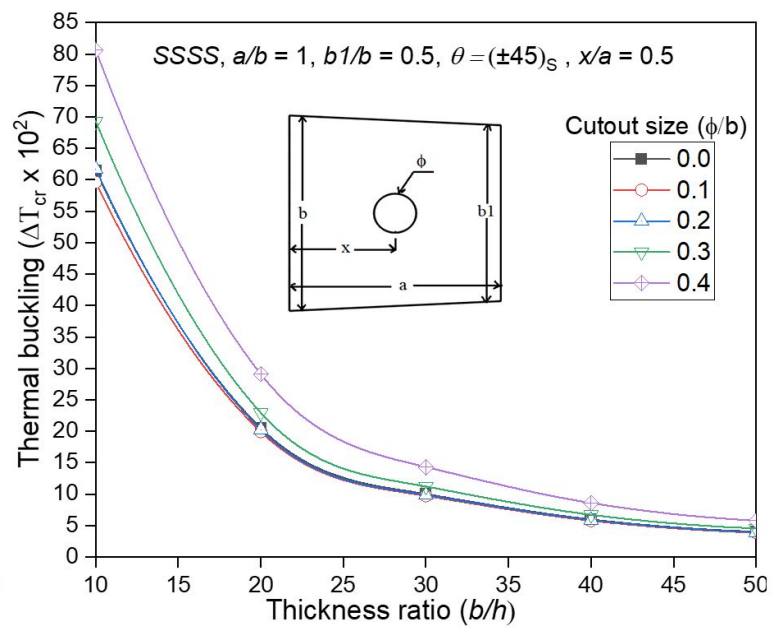

(b)

Figure 6 Thermal buckling behaviour under different thickness a) cross-ply and (b) angle-ply.

\subsubsection{Impact of plate aspects and trapezoidal shapes on thermal buckling behaviour.}

Effect of different plate aspect ratios and the trapezoidal shapes on the thermal buckling behaviour of the trapezoidal plate have been investigated in this section and the results are illustrated in Figure 7 by considering simply supported boundary conditions. The size of the circular hole which is considered here is 0.2 and having the thickness ratio $(b / h)=100$. Both cross ply and the angle ply laminated scheme are considered in order to compare the outcome the analysis. It is observed from the study that as the plate aspect ratio increases, the thermal buckling decrease and found to be maximum when the $a / b=1$. It is also observed from the study that, as the trapezoidal shape ratio increases, the thermal buckling stability will also be increased and is found to be minimum when $b_{1} / b$ is 1.0 . However, the thermal buckling resistance is quite higher in case of angle ply laminated scheme as compared with the cross ply laminated scheme as shown in Figure 7 (a) and Figure 7 (b). Moreover trapezoidal shape with trapezoidal shape ratio $\left(b_{1} / b\right) 0.5$ shows the higher critical thermal buckling resistivity as compared with the square/rectangular laminated plate at simply supported boundary condition. It is also noting from Figure 7 (a) and Figure 7 (b) that, the thermal buckling stability is found to be maximum in case of angle-ply composite laminates at plate aspect ratio $(a / b)$ is 1.0.

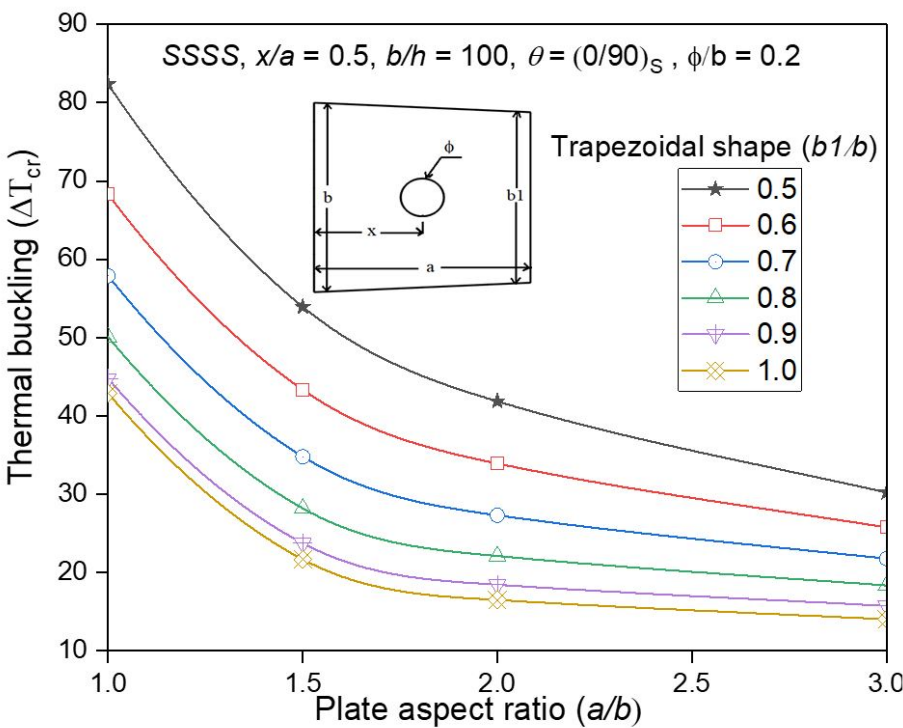

(a)

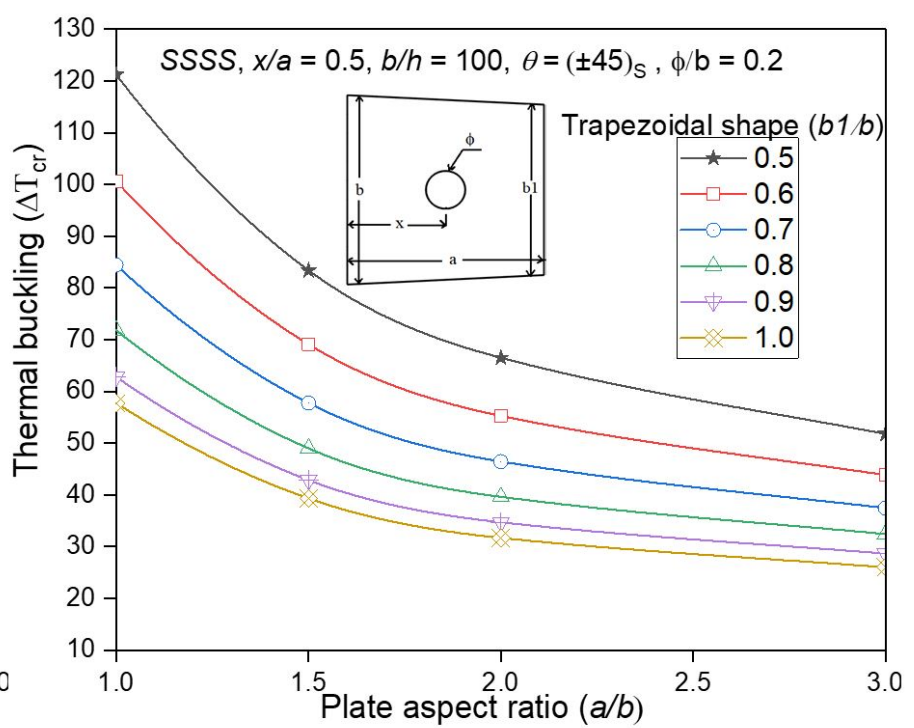

(b)

Figure 7 Thermal buckling behaviour under plate aspect ratio (a) cross ply and (b) angle ply laminates. 


\subsubsection{Impact of different edge Conditions on thermal buckling behaviour.}

Influence of different edge conditions and the trapezoidal shapes on the thermal buckling behaviour of laminated plate has been studied by considering a cutout offset ratio of 0.5 with different trapezoidal shape ratios. To analyse the thermal buckling behaviour under different edge conditions, two set of ply-configurations are considered i.e cross-ply and angle-ply layup schemes. The responses of the study are shown in the Figure 8 (a). It is observed from Figure 8 (a) and Figure $8(\mathrm{~b})$ that the buckling resistance of trapezoidal plate remains unchanged as the trapezoidal shape ratio $\left(b_{1} / b\right)$ increases irrespective of the edge conditions. The highest thermal bucking stability is found in case of cross ply laminated scheme at CCFF edge condition as shown in Figure 8 (a). However, for the same ply orientation the minimum thermal buckling stability is found at SSSS edge condition with $b_{1} / b=1.0 \mathrm{It}$ is also observed from the Figure 8 (b) that for any given boundary condition, the value of $\Delta T_{c r}$ initially higher at $C C C C$ edge condition and reduces as the $b_{1} / b$ ratio increases uniformly. Further, the lowest thermal buckling resistivity is found for SSSS boundary conditions in angle ply laminates. In over all, it is concluded from the study that, the thermal buckling stability is found highest in case of cross ply laminated scheme with same edge conditions.

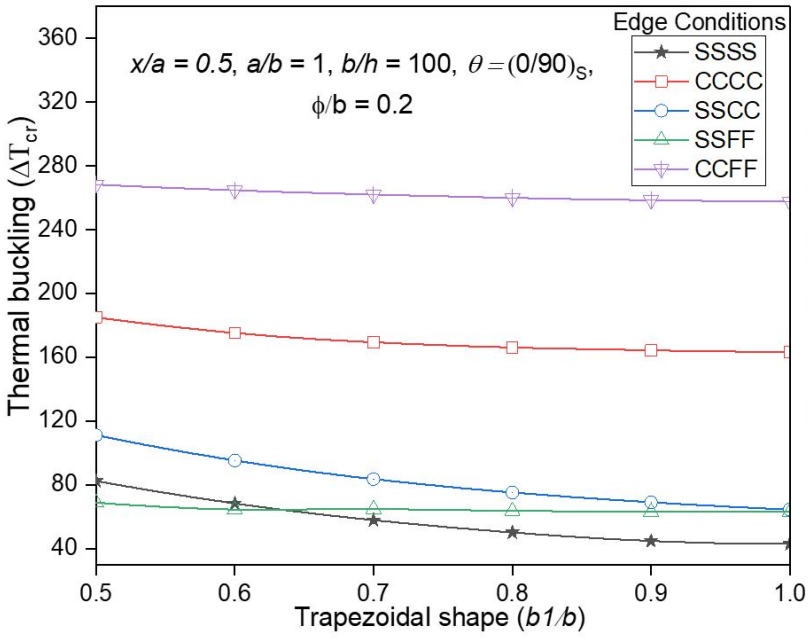

(a)

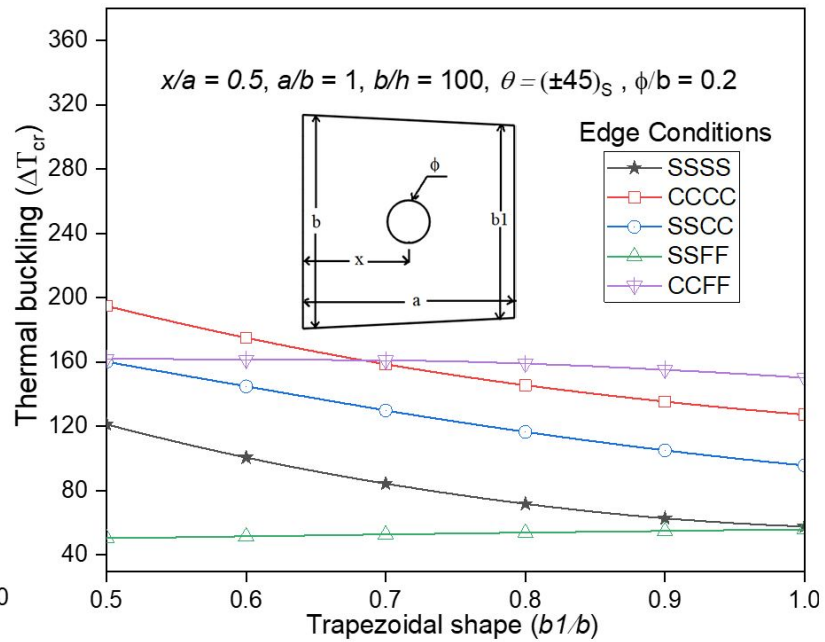

(b)

Figure 8 Thermal buckling behaviour under different edge conditions (a) cross ply laminate (b) angle ply laminates

\section{CONCLUSIONS}

Thermal buckling behaviour of trapezoidal plates with different sized cutouts have been studied in this research by considering different trapezoidal shapes with uniformly temperature rise. The important findings from this work are as follows:

- For any given trapezoidal shape ratio and uniformly temperature rise, the thermal buckling resistance of the plate increases with the increase in ply-orientation and found to be highest at an angle of $( \pm 45)$ s for both simply supported and clamped edge conditions.

* The critical thermal buckling depends heavily on the size of the cutout. As the cutout sizes increases, thermal buckling resistance increases and found be maximum at higher cutout size.

* The trapezoidal shapes play a significant role on the thermal buckling resistance. For any given cutout size, the thermal buckling resistance of the plate increases with the increase in trapezoidal shapes and found to be highest at $b 1 / b=0.5$.

* The thermal buckling stability decreases gradually when cutout move towards the higher stiffness zones. However, this behaviour is same in cross ply laminates, and not found much difference in the thermal buckling stability as the cutout offsets.

* As the thickness decreases, the laminated plate becomes thin, and hence reduces the strength. This leads to decrease in the stability of the laminated plate.

* It is observed from the study that as the plate aspect ratio increases, the thermal buckling decrease and found to be maximum when the $a / b=1$. 
* The highest thermal bucking stability is found in case of cross ply laminated scheme at CCFF edge condition for the same ply orientation the minimum thermal buckling stability is found at SSSS edge condition.

Author's Contribution: Conceptualization: Maharudra, T Rajanna and Bheemsha Arya; Methodology: T Rajanna; Investigation: Maharudra; Writing original draft: Maharudra; Writing review \& editing: T Rajanna and Bheemsha Arya; Supervision: T Rajanna and Bheemsha Arya.

Editor: Marco L. Bittencourt.

\section{REFERENCES}

Biswal, M., Sahu, S,Asha, A. (2016). Vibration of composite cylindrical shallow shells subjected to hygrothermal loadingexperimental and numerical results, Composites Part B: Engineering 98: 108-119.

Rath,M.K., Sahu,S.K. (2013). Parametric Instability of square laminated Plates in hygrothermal environment, Journal of Structures .

H. Panda, S. Sahu, P. Parhi. (2013). Hygrothermal effects on free vibration of delaminated woven fiber composite platesNumerical and experimental results, Composite Structures 96: 502-513.

Vishesh R. Kar., Subrata, K., Panda and Trupti R. Mahapatra. (2016). Thermal buckling behaviour of shear deformable functionally graded single/doubly curved shell plate with TD and TID properties Advances in Materials Research 5 (4): $205-221$

Na, K.S. and Kim, J.H. (2004). Three-dimensional thermal buckling analysis of functionally graded materials, Compos. Part BEng., 35(5): 429-437.

Abdelhak, Z., Hadji, L., Daouadji, T.H. and Bedia, E.A. (2015). Thermal buckling of functionally graded plates using a n-order four variable refined theory, Adv. Mat. Res. 4(1): 31-44.

Ashraf M. Zenkour. (2012). Hygrothermal effects on the bending of angle-ply composite plates using a sinusoidal theory. Composite Structures 94: 3685-3696.

Sai Ram, K. S and Sinha, P. K. (1991). Hygrothermal effects on the bending characteristics of laminated composite plate. Int. Journal of computers and structures 40(4): 1009-1015.

Shen, H.S.(2001). Hygrothermal effects on the post-buckling of shear deformable laminated plates. Int J Mech Sci 43: $1259-81$.

Marwa Abida., Florian Gehringa., Jamel Marsb., Alexandre Viveta., Fakhreddine Dammakb., Mohamed Haddarc. (2020). Hygromechanical coupling and multiscale swelling coefficients assessment of flax yarns and flax / epoxy composites. Composites Part A 136: 105914 .

Abdelmalek Abdelmalek., Mokhtar Bouazza., Mohamed Zidour., Noureddine Benseddiq. (2017). Hygrothermal effects on the free vibration behaviour of composite plate using nth-order shear deformation theory: a micromechanical approach. Iran J Sci Technol Trans Mech Eng.

Debabrata Gayen., Tarapada Roy. (2013). Hygro-thermal effects on stress analysis of tapered laminated composite beam. International Journal of Composite Materials 3(3): 46-55.

Madhusmita Biswal., Shishir, K.R. Sahu, A.V., Asha and Namita Nanda. (2016). Hygrothermal effects on buckling of composite shell-experimental and FEM results. Steel and Composite Structures 22(6): 1445-1463.

Patel, B. P, Ganapathi, M, Makhecha, D.P. (2002). Hygrothermal effects on the structural behaviour of thick composite laminates using higher-order theory. Composite Structures 56: 25-34.

Omer Sinan Sahin. (2005). Thermal buckling of hybrid angle-ply laminated composite plates with a hole. Composites Science and Technology 65: 1780-1790.

Abdelbaki Chikh, Abdelouahed Tounsi, Habib Hebali and Mahmoud, S. R. (2017). Thermal buckling analysis of cross-ply laminated plates using a simplified HSDT. Smart Structures and Systems 19(3): 289-297.

Chandrashekhara, K. (1992). Thermal buckling of laminated plate using a shear flexible finite element, finite elements in analysis and design 12: 51-61. 
Shu, X.P., Sun, L.X. (1994). Thermo-Mechanical Buckling of Laminated Composite Plates With Higher Order Transverse Shear Deformation. Computers and Structures 53(1): 1-7.

Mansouri, M.H., Shariyat, M. (2014). Thermal buckling predictions of three types of high-order theories for the heterogeneous orthotropic plates, using the new version of DQM. Composite Structures 113: 40-55.

Ghannadpour, SAM., Ovesy, H.R., Nassirnia M. (2012). Buckling analysis of functionally graded plates under thermal loadings using the finite strip method. Computers \& Structures 108-109: 93-99.

Chen, C.S., Lin, C.Y., Chien, R.D. (2011). Thermally induced buckling of functionally graded hybrid composite plates. International Journal of Mechanical Sciences 53(1): 51-58.

Shariyat. (2007). Thermal buckling analysis of rectangular composite plates with temperature-dependent properties based on a layerwise theory. Thin-Walled Structures 45: 439-452.

Noor, A.K., Burton, W.S. (1992). Three-dimensional solutions for thermal buckling of multilayered anisotropic plates. J Eng Mech 118 (4): 683-701.

Carrera, E. (2005). Transverse normal strain effects on thermal stress analysis of homogeneous and layered plates. AIAA Journal 43(10): 2232-2242.

Bathe., Klaus-Jurgen. (2014). Finite elements procedures. Second Edi. Prentice Hall, Pearson Education, Inc.

Maharudra., Arya, B., Rajanna, T. (2020). Effect of ply-orientation and boundary conditions on the vibrational characteristics of laminated composite plates using HOSDT, Mater. Today: Proc. 20: 134-139.

Rajanna, T., Sauvik Banerjee., Yogesh, M. Desai., Prabhakara, D. L. (2016). Effects of Partial edge loading and fibre configuration on vibration and buckling characteristics of stiffened composite plates. Latin American Journal of Solids and Structures 13: 854-879.

Yathish Muddappa, P.P., Rajanna, T., Giridhara, G. (2021). Effects of different interlaminar hybridization and localized edge loads on the vibration and buckling behaviour of fiber metal composite laminates. Composites Part C 4: 100084.

Subash Chandra, K. S., Rajanna, T., Venkata Rao, K. (2020). A Parametric study on the effect of elliptical cutouts for buckling behaviour of composite plates under non-uniform edge loads. Latin American Journal of Solids and Structures 17: 1-15. 